\title{
Practice \& SWOT Analysis of Drop-and-Pull Transportation Mode in Ningbo City
}

\author{
Yi Han ${ }^{1}$, Hexin Lyu², Pengfei Pan³, Guoyong Dai ${ }^{4}$, Shuting Bi ${ }^{5}$, Liyan Wang6 \\ ${ }^{1}$ College of Jiayang, Zhejiang Shuren University, Hangzhou, China \\ ${ }^{2}$ College of Innovation and Entrepreneurship, Zhejiang Shuren University, Hangzhou, China \\ ${ }^{3}$ School of Artificial Intelligence and Computer Science, Changzhou University, Changzhou, China \\ ${ }^{4}$ College of Information Science and Technology, Zhejiang Shuren University, Hangzhou, China \\ ${ }^{5}$ Aviation Service Institute, Jiangsu Aviation Technical College, Zhenjiang, China \\ ${ }^{6}$ School of Information and Control Engineering, Liaoning Petrochemical University, Fushun, China \\ Email: *187267654@qq.com
}

How to cite this paper: Han, Y., Lyu, H. X., Pan, P. F., Dai, G. Y., Bi, S. T., \& Wang, L. Y. (2022). Practice \& SWOT Analysis of Drop-and-Pull Transportation Mode in Ningbo City. Journal of Service Science and Management, 15, 1-9.

https://doi.org/10.4236/jssm.2022.151001

Received: December 6, 2021

Accepted: January 11, 2022

Published: January 14, 2022

Copyright (c) 2022 by author(s) and Scientific Research Publishing Inc. This work is licensed under the Creative Commons Attribution International License (CC BY 4.0).

http://creativecommons.org/licenses/by/4.0/

(c) (i) Open Access

\begin{abstract}
Entering the 21st century, the world's road transportation industry has been developing rapidly. Many cargos were loaded and transshipped by road from one side of a country to the other side, instead of staying on boats, to save time. However, road transportation has been facing heavy pressures like high energy consumptions and pollution emissions. Therefore, an efficient and lowemission transportation mode, drop-and-pull (DP), emerged and was widely acknowledged all over the world. China's Ministry of Transport started a pilot project of DP transportation in 2010. Several provinces and cities, including Ningbo City in Zhejiang Province, across the country were selected as pilot zones for DP transportation. Here, the practice of DP transportation mode in Ningbo is clearly depicted according to a dozen of logistics and shipping companies' activities and an all-round SWOT analysis about Ningbo city is fully presented as a valuable reference for decision makers to precisely grip Ningbo's advantages and further tailor Ningbo to suit the DP transportation mode better.
\end{abstract}

\section{Keywords}

Drop-and-Pull, Road Transportation, Shipping, SWOT, Cargo

\section{Introduction}

Road transportation, which is an important part of modern logistics system, plays an important role in supporting and guaranteeing the country's economic and social development. Improving the efficiency of transportation vehicles by 
optimizing the organization and management methods is the most convenient and efficient way to realize the upgrading and transformation throughout the whole industry. Drop-and-Pull (DP) mode is a key mean to reach this goal. In DP, a prime mover truck sends a container trailer from one site to a destination and drops the trailer there. Then after a short period of layover time, the truck hit the road again towing another trailer to its destination (Dong, 2011; Epeterson, 2000; Gerdessen, 1996; Liu \& Lin, 2011; Manfred \& Hartl, 2003; Qi \& Ma, 2012; Westerlund, 2009).

\section{Development of DP in Ningbo}

Ningbo is an important core area of maritime economy in China with obvious geographical and regional advantages. In recent years, Ningbo has successively completed the construction of expressway network reaching six economical metropolises. In terms of road transportation, 4 important logistics areas-Beilun, Zhenhai, Meishan and Airport have been created. And a logistics park with freight stations has been formed. More than 600 ports in more than 100 countries and regions worldwide have established international shipping cooperation with Ningbo with more than 200 shipping lines and 800 freight flights per month. Beilun Port, the second largest deep-water port in China, has ample capacity to support large throughput of bulk commodities and containers and transship them with container vehicles. Nowadays, there are $8 \mathrm{DP}$ companies as listed in Table 1.

In 2010, Ningbo, which is an important port city and logistics hub on the east coast in China, was designated as a pilot zone for DP transportation in Zhejiang Province. Since then, according to recent statistics, many shipping companies there have upgraded their fleet to owing more than 430 prime mover trucks and 700 container trailers. Moreover, they opened up a large amount of DP transportation routes, thus enrich the DP transportation network system (Statistics Data, 2021).

Table 1. DP logistics companies.

\begin{tabular}{c} 
Company Names \\
\hline Zhejiang Baifu Logistics Co., Ltd. \\
Ningbo Lingyu and Logistics Co., Ltd. \\
Ningbo Hengsheng Logistics Co., Ltd. \\
Ningbo Port Container Transport Co., Ltd. \\
Ningbo Fubon Logistics Co., Ltd. \\
Ningbo Wenchang Int. Logistics Co., Ltd. \\
Ningbo Zhongtong Logistics Co., Ltd. \\
Cixi Highway-Rail Combined Trans Co., Ltd.
\end{tabular}

Source: Statistics Data from Transportation Bureau of Zhejiang Province 2021. 
With the rapid development of Ningbo's local economy, the main body of Ningbo's road transportation market has ever growing and developing. There are more than 49,512 road freight operators, 507 container transportation companies, 12,554 container vehicles and 4500 logistics service companies engaging in freight forwarding, warehousing services and professional transportation in Ningbo. Furthermore, the Ningbo Municipal Government has also made great efforts to support a large number of local logistics companies in the Yangtze River Delta region and abroad, like Ningbo COSCO Logistics, Zhejiang Sinotrans, Zhongtong, Hailian, Jinxing etc. Some multinational shipping giants like DHL, MAERSK and MSC have already footed in Ningbo (Statistics Data, 2021).

\section{DP Facilities and Equipment}

\subsection{DP Stations}

Zhejiang Baifu Logistics Co. Ltd. has completed a total investment of 118 million yuan to bring Shaoxing Jiya Logistics park into operation. In addition, Baifu established a DP operation center within Beilun Port to rebuilt and expand the DP loading \& unloading site and a parking lot. Ningbo Lingyu Logistics has invested 11.26 million yuan on its station in Xiapu International Logistics Park. Thus a new operation platform taking up 1250 square meters for road-rail combined transport was created. Zhongtong Logistics invested more than 800,000 yuan to flat the ground of its logistics park (Feng, 2013; Han, 2012; Li \& Zhou, 2013; Peng, Zhong, \& Huang, 2013; Zhang, Liu, \& Zhou, 2013; Zhao, 2013).

\subsection{DP Vehicles}

Since the launch of DP, those pilot logistics enterprises have purchased 427 DP trucks and 678 container trailers. Table 2 shows the detailed number of DP vehicles in each pilot enterprise.

\subsection{Information Platform}

Ningbo Lingyu Logistics adopted a "Container Logistics Service Platform", which used an open B2B logistics information operational mode to realize the automatic handling of DP business. This improved the scientific dispatching ability of DP vehicles and realized information sharing with membership shipping companies (Statistics Data, 2021).

Baifu and Fubang have invested millions of dollars respectively to establish intelligent logistics information platforms to realize the routinization, standardization and visualization of internal traffic management. And set up an interactive communication channel with customers. The two companies also integrated information technologies such as GPS, dynamic video, business synchronization and message sending into logistics software enabling their dispatching management, transportation management, warehouse management, loading \& unloading management, order management and station monitoring (Statistics Data, 2021). 
Table 2. DP vehicles employed by 8 companies.

\begin{tabular}{cccc}
\hline Company Name & Trucks & Trailers & Total \\
\hline Zhejiang Baifu Logistics Co., Ltd. & 60 & 100 & $\mathbf{2 0 1}$ \\
Ningbo Port Ling and Logistics Co., Ltd. & 91 & 111 & $\mathbf{9 5}$ \\
Ningbo Hengsheng Logistics Co., Ltd. & 35 & 49 & 96 \\
Ningbo Port Container Transport Co., Ltd. & 127 & 243 & $\mathbf{4 7 2}$ \\
Ningbo Fubon Logistics Co., Ltd. & 60 & 80 & 106 \\
Ningbo Wenchang Int. Logistics Co., Ltd. & 30 & 36 & 50 \\
Ningbo Zhongtong Logistics Co., Ltd. & 9 & 36 & $\mathbf{6 2}$ \\
Cixi Highway-Rail Combined Trans Co., Ltd. & 15 & 23 & 150 \\
Total Number of Vehicles & 427 & $\mathbf{6 7 8}$ & $\mathbf{1 1 0 5}$ \\
\hline
\end{tabular}

Source: Statistics data from Transportation Bureau of Zhejiang Province 2021.

\subsection{DP Operation}

So far, most DP companies in Ningbo City have been following one operation mode, in which several DP stations in different cities were serialized in one route. When a truck reached a station, it would tow another trailer to another station with only a short interval (Zheng, 2012; Ning, 2013).

Zhongtong Logistics adopted "one line with two points" operation mode. The advantage of this method is to maximize the utility rate of DP vehicles, speed up the freight turnover rate and save the loading and unloading time. Fubon Logistics and Lingyu Logistics have mainly adopted "one line with multiple points" mode. A truck sets out from the first city to another city. When the truck reaches each city, the container trailer is dropped and another pre-loaded trailer is pulled to the next city. The process is repeated until the truck returns to the first city in a closed transportation loop (Statistics Data, 2021).

\section{Benefits of DP Transportation}

DP transportation reduced costs for logistics companies. The DP mode, compared with the traditional container trucks transportation mode, only needs several prime mover trucks which can be separated from the trailers. Thus the number of trucks required is far less than the number of traditional container trucks. This point can be easily envisioned like if we only purchased several trucks and dozens of trailers, we can start the transportation operation by keeping those trailers in those DP stations for loading \& unloading activities while making trucks to drop and pull trailers at stations. This reduction in the number of trucks indirectly led to the reduction of labor costs and vehicle maintenance costs. Moreover, DP mode increased the turnover of trucks and brought about a reduction in unit transportation cost.

Due to the improved operation efficiency, the fuel consumption per 100 ton-kilometer has dropped by 0.6 liters. Assuming that a company has 100 trucks, 
it can save 3,837,240 liters of diesel, which is equivalent to 4808 tons of standard coal, a year (Cai, 2012; Li, 2012).

Ningbo Port Container Transport Company, Wenchang Int. Logistics and Cixi Road-rail Transport Company have increased their investment in energy and environmental protection during the practice of DP transportation. LNG clean energy trucks were purchased to realize modern low-consumption and high-efficiency operational mode (Statistics Data, 2021).

\section{SWOT of DP Mode in Ningbo}

\subsection{Strengths}

Ningbo is located at the mouth of China's Yangtze River Delta and is a strategically important foreign trade center in the whole area. Ningbo Beilun Port is China's second largest deep-water port and the largest transshipment port for international iron ore transportation. Ningbo is also a major transshipment, import and export port for coal, crude oil and container transportation in East China. The Hangzhou Bay Bridge connects Ningbo, Shanghai, Hangzhou, Nanjing and other economic cities in the Yangtze River Delta and especially creates a two-hour traffic circle between Ningbo and international metropolis-Shanghai. In recent years, with the accelerated implementation of Ningbo's port-bridgeand-sea linking strategy, Ningbo's economy agglomeration capacity and external proliferation capacity have been continuously enhanced.

Ningbo's shipping infrastructure construction has made remarkable achievements, and Ningbo has become an important transportation hub city in East China. Ningbo Port has established links with 600 ports in 100 countries and regions around the world, with more than 200 routes and more than 800 international shipping freighters (Statistics Data, 2021).

Ningbo Lishe Airport has become an important zone with more than 50 domestic and foreign air routes directly reaching Bangkok, Tokyo and Seoul. Furthermore, Ningbo City has built a highway network with the "One loop with 6 cities" highway as the main route. Ningbo has also opened many high-speed railways going to Beijing, Shanghai, Wuhan, Guangzhou and metropolitans alike (Statistics Data, 2021).

Ningbo port has abundant bulk cargo and container freight resources. Sufficient cargo sources meet the requirements of DP transportation. Ningbo Port holds cargo throughput of 384 million tons per year, which makes it rank second among domestic ports and fourth worldwide. And it could complete container throughput of 10.423 million TEUs ranking 8th in China and around the globe. Over $90 \%$ of the containers transportation in Ningbo Port is carried out by DP transportation mode now (Statistics Data, 2021).

\subsection{Weaknesses}

Those who enter the field of DP transportation are all large-scale transportation companies with a certain scale. That means it is difficult for small and medium- 
sized transportation companies to enter this transportation market. Because entering the DP transportation market requires huge investment in the early stage to purchase transportation vehicles, build stations and establish other infrastructures. The return on the capital invested could not be obtained in a short time.

The policies related to DP transportation did not take into account the needs of DP at the beginning of DP's birth, which resulted in a disjoined development. Currently, Policies for the introduction of trailer inspection system, trailer license plate management system and customs supervision system, etc. are still in the incubator.

Compared with traditional mode, DP transportation is a more refined operation method, which requires the connection of all links of transportation to be kept smooth. So it has a great requirement for higher timeliness for the scientific dispatch of vehicles, cargo and loading \& unloading operations. However, most of the shipping enterprises are still lacking information processing capacity, which hindered the fast and healthy development of DP transportation.

In addition, the overall quality of the employees in DP transportation industry is not high. The personnel team is unable to meet the growing demand for optimal DP operation. Therefore, there is still some gap to bridge before DP transportation is fully deployed in Ningbo.

\subsection{Opportunities}

Drop-and-Pull transportation is an important way to achieve the strategic goals of resource-saving and environment-friendly. Supporting policies has been enforced by the local government. The Ningbo Municipal Government has attached great importance to the development of DP transportation, trying to forge Ningbo into a modern logistics zone.

In recent years, the Ningbo Municipal Government has vigorously changed the economic growth mode and promoted economic restructuring. In order to meet the needs of economic development, the transformation and upgrading of the road freight industry has become more realistic and urgent. At the same time, with the strengthening of China's efforts to control overloading, safe production, and environmental protection, the previous transportation mode has gradually been replaced by a development mode that improved transportation efficiency, forced the Ningbo road freight market to go towards integration, intensification, intelligence, and efficiency.

\subsection{Threats}

In order to ensure the healthy operation of DP model, and to further improve the transportation efficiency and service quality, enterprises must deepen the transformation of the existing DP vehicles and stations. This requires companies to invest huge money to purchase DP trucks and trailers, upgrade transportation stations and upgrade DP information systems, etc. For example, enterprises can only rely on self-raise funds and bank loans to update vehicles and loading \& 
unloading equipment. However, with shortened vehicle elimination cycle, higher vehicle depreciation rate and bank loan interest, companies will shoulder higher operating costs.

As an advanced and scientific transportation model, DP transportation is still in its early stage. Most customers are still accustomed to traditional transportation methods, which results in a slow development of DP transportation. In addition, due to the limited knowledge of DP transportation, the market cultivation period for each DP route is longer.

In recent years, with the continuous increase of employees' wages, tax costs, and oil prices, it has become a major challenge for the operation and development of DP transportation companies. Among them, taxation and oil price costs are major problems that plague DP companies.

\section{Analysis on DP Mode in Ningbo City}

Through the use of internal and external factor evaluation matrices (IFE matrix and EFE matrix) (Huang, 2008), we can summarize, analyze and evaluate the economic environment, social environment, political environment and other aspects that affect the development of Ningbo DP transportation. The steps to establish IFE matrix and EFE matrix are as follows:

1) List the internal and external evaluation factors for DP transportation, and invite some experts, industry managers, and business leaders to give scores for each factor.

2) According to the SWOT analysis method, each unimportant factor weight value is 0 , and each important factor weight value is 1 .

3) Score each key factor according to its strength. The strength is divided into seven levels with values of $-3,-2,-1,0,1,2$, and 3 respectively. Among them, the strength values of opportunities and advantages are positive numbers, and the strength values of threats and disadvantages are negative numbers. The greater their absolute value, the greater their intensity.

4) By multiplying the score of each factor by its corresponding weight, we can get the weighted score of each factor. Then the IFE matrix and EFE matrix are obtained as shown in Table 3 and Table 4.

The influence degree of a certain factor is equal to the average weight multiplied by the evaluation score. Through the following formula, we can know the total strength of each aspect. Total Strength $=\sum S_{i} *$ score $=1.26$, total strength of Weakness $=\sum W_{i} *$ score $=-0.76$, Total opportunity $O=\sum O_{i} *$ score $=$ 1.35 and total threat $T=\sum T_{i} *$ score $=-0.96$.

According to a formula $\operatorname{tg} \theta=(1.26-0.76) /(1.35-0.96)=1.28$, we can get $\theta=$ $52^{\circ}$ by using inverse trigonometric function. The strategic angle $\theta$ of DP transportation development prospect in Ningbo is between $[\pi / 4, \pi / 2]$ in the first quadrant. As a result, According to Table 5, Ningbo's DP transportation development should be based on the characteristics of port economy, sources of goods, scale of transportation, and technical and information-based transformation. 
Table 3. Evaluation of internal factors of industry development.

\begin{tabular}{ccccc}
\hline Aspects & Factors & Average & Score & Total \\
\hline \multirow{4}{*}{ Strengths } & $S_{1}$ good location advantage & 0.16 & 2 & 0.32 \\
& $S_{2}$ transportation infrastructure & 0.17 & 2 & 0.34 \\
& $S_{3}$ sufficient freight demand & 0.14 & 3 & 0.42 \\
& $S_{4}$ growing road transportation market & 0.18 & 1 & 0.18 \\
\hline \multirow{3}{*}{ Weaknesses } & $W_{1}$ high investment cost & 0.16 & -3 & -0.48 \\
& $W_{2}$ institutional barriers & 0.07 & -1 & -0.07 \\
& $W_{3}$ management \& tech. backwardness & 0.09 & -2 & -0.18 \\
& $W_{4}$ professional and technical personnel & 0.03 & -1 & -0.03 \\
\hline & total & 1 & & 0.5
\end{tabular}

Source: Based on Expert Interviews.

Table 4. Evaluation of external factors in industry development.

\begin{tabular}{ccccc}
\hline Aspects & Factors & Average & Score & Total \\
\hline \multirow{4}{*}{ Opportunities } & $O_{1}$ The introduction of relevant policies & 0.12 & 2 & 0.24 \\
& $O_{2}$ support from local governments & 0.17 & 3 & 0.51 \\
& $O_{3}$ Opportunities for the transformation & 0.15 & 2 & 0.3 \\
& $O_{4}$ Energy and emission reduction & 0.15 & 2 & 0.3 \\
\hline \multirow{3}{*}{ Threats } & $T_{1}$ funding shortage & 0.14 & -3 & -0.42 \\
& $T_{2}$ incubation period of the market & 0.15 & -2 & -0.3 \\
& $T_{3}$ operating costs & 0.12 & -2 & -0.24 \\
\hline & Total & 1 & & 0.39 \\
\hline
\end{tabular}

Source: Based on Expert Interviews.

Table 5. Strategies and types of DP's development in ningbo.

\begin{tabular}{|c|c|c|c|c|c|c|c|}
\hline \multicolumn{2}{|c|}{ Quadrant I } & \multicolumn{2}{|c|}{ Quadrant II } & \multicolumn{2}{|c|}{ Quadrant III } & \multicolumn{2}{|c|}{ Quadrant IV } \\
\hline Pioneering & trategy & Struggli & trategy & Conservativ & e strategy & Resista & Strategy \\
\hline type & range & type & range & type & range & type & range \\
\hline Potential & $0, \pi / 4$ & Aggres & $2,3 \pi$ & Withdrawl & $\pi, 5 \pi / 4$ & Adjus & $3 \pi / 2,7 \pi / 4$ \\
\hline
\end{tabular}

Source: Based on Huang, 2008.

\section{Discussion}

This paper described a brief development history of DP transportation mode in Ningbo City since 2010. According a SWOT analysis on DP mode, the overall development direction was suggested. Thus, a constructive guideline was presented to help those decision makers to adopt targeted measures for better promoting the development of DP transportation in Ningbo City.

\section{Fund Project}

Zhejiang Shuren University Talent Project KXJ1420601. Zhejiang Public Research Foundation Project LGF18F020009. 


\section{Conflicts of Interest}

The authors declare no conflicts of interest regarding the publication of this paper.

\section{References}

Cai, F. (2012). Current Situation Evaluation and Trend Analysis of Traffic Energy Conservation and Emission Reduction. Traffic Energy Conservation and Environmental Protection, 1, 1-3.

Dong, H. (2011). Research on the Production Organization Mode of Road Transportation. Chang'an University.

Epeterson, B. (2000). Intermodal and International Freight Network Modeling. Transportation Research Part C: Emerging, 8, 147-166.

Feng, J. (2013). Discussion on Promoting the Organization Mode of Road Freight Transportation in Shandong Province. Foreign Investment in China, 7, 268-269.

Gerdessen, J. C. (1996). Vehicle Muting Problem with Trailers. European Journal of Operational Research, 93, 135-147.

Han, L. (2012). Semi-Trailer Efficient Turnover Technology and Market Analysis. Logistics Technology and Application, 4, 26-28.

Huang, R. (2008). SWOT Quantitative Measurement Model Based on Coordinate Method and Its Application Research. Scientific Research Management, 1, 179-187.

Li, C., \& Zhou, A. (2013). Analysis on the Prospect of the Development of Transportation in the Pearl River Delta Region. Highway and Motor Transport, 1, 76-79.

Li, D. (2012). Research on Wuhai Railway Freight Development Strategy Based on SWOT Analysis. Chang'an University.

Liu, D., \& Lin, Q. (2011). Transport Benefits and Paths of Road Transport Enterprises Development. Journal of Fujian University of Technology, 9, 616-620.

Manfred, G., \& Hartl, R. F. (2003). New Saving Based Algorithm for Time Constrained Pickup and Delivery of Full Truckloads. European Journal of Operational Research, 151, 520-535.

Ning, W. (2013). How to Realize Energy-Saving Transportation by Trailer. Special Purpose Vehicle, 2, 70-72.

Peng, X., Zhong, P., \& Huang P. (2013). Study on the Construction and Development of the Transportation System in Guangxi. Highway and Bridge, 14, 78-80.

Qi, H., \& Ma, Y. (2012). Research on the Optimization Model of Low-Carbon Transportation Organization Based on Drop-and-Drop Transportation. Logistics Engineering and Management, 32, 111-115.

Statistics Data (2021). Transportation Bureau of Zhejiang Province. http://jtyst.zj.gov.cn

Westerlund, K. R. (2009). Feasibility of Longer Combination Vehicles-A Pre-Study of Longer Combination Vehicles in Hub to Hub Long Haulage of Mixed Goods. Charmers University of Technology.

Zhang, X., Liu, M., \& Zhou, G. (2013). Study on the Standard System of Highway Transportation. Communications Standardization, 9, 8-11.

Zhao, J. (2013). Discussion on the Present Situation and Countermeasures of Drop-Off Transportation in Henan Province. Journal of Henan Electromechanical College, 2, 3538.

Zheng, Y. (2012). Conditions and Operating Rules of Semi-Trailer Transport Trains. Commercial Vehicles, 4, 67-68. 